\title{
Chromatic number for a generalization of Cartesian product graphs
}

\author{
Daniel Král ${ }^{*}$ \\ Institute for Theoretical Computer Science \\ Faculty of Mathematics and Physics \\ Charles University, Prague, Czech Republic \\ kral@kam.mff.cuni.cz
}

\author{
Douglas B. West ${ }^{\dagger}$ \\ Mathematics Department \\ University of Illinois, Urbana, IL \\ west@math . uiuc . edu
}

Submitted: Aug 1, 2008; Accepted: Jun 7, 2009; Published: June 19, 2009

Mathematics Subject Classification: 05C05

\begin{abstract}
Let $\mathcal{G}$ be a class of graphs. A $d$-fold grid over $\mathcal{G}$ is a graph obtained from a $d$-dimensional rectangular grid of vertices by placing a graph from $\mathcal{G}$ on each of the lines parallel to one of the axes. Thus each vertex belongs to $d$ of these subgraphs. The class of $d$-fold grids over $\mathcal{G}$ is denoted by $\mathcal{G}^{d}$.

Let $f(\mathcal{G} ; d)=\max _{G \in \mathcal{G}^{d}} \chi(G)$. If each graph in $\mathcal{G}$ is $k$-colorable, then $f(\mathcal{G} ; d) \leq k^{d}$. We show that this bound is best possible by proving that $f(\mathcal{G} ; d)=k^{d}$ when $\mathcal{G}$ is the class of all $k$-colorable graphs. We also show that $f(\mathcal{G} ; d) \geq\left\lfloor\sqrt{\frac{d}{6 \log d}}\right\rfloor$ when $\mathcal{G}$ is the class of graphs with at most one edge, and $f(\mathcal{G} ; d) \geq\left\lfloor\frac{d}{6 \log d}\right\rfloor$ when $\mathcal{G}$ is the class of graphs with maximum degree 1.
\end{abstract}

\section{Introduction}

The Cartesian product of graphs $G_{1}, \ldots, G_{d}$ is the graph with vertex set $V\left(G_{1}\right) \times \cdots \times$ $V\left(G_{d}\right)$ in which two vertices $\left(v_{1}, \ldots, v_{d}\right)$ and $\left(v_{1}^{\prime}, \ldots, v_{d}^{\prime}\right)$ are adjacent if they agree in all but one coordinate, and in the coordinate where they differ the values are adjacent

${ }^{*}$ The Institute for Theoretical Computer Science (ITI) is supported by the Ministry of Education of the Czech Republic as project 1M0545. This research was also partially supported by the grant GACR 201/09/0197.

${ }^{\dagger}$ Research partially supported by the National Security Agency under Award No. H98230-06-1-0065. 
vertices in the corresponding graph. The product can be viewed as a rectangular grid with copies of $G_{1}, \ldots, G_{d}$ placed on vertices forming lines parallel to the $d$ axes. It is well-known (and easy to show) that the chromatic number of the Cartesian product of $G_{1}, \ldots, G_{d}$ is the maximum of the chromatic numbers of $G_{1}, \ldots, G_{d}[12]$.

In this paper, we consider bounds on the chromatic number of graphs in a family resulting from a more general graph operation. Instead of placing copies of the same graph $G_{i}$ on all the lines parallel to the $i$-th axis, we may place different graphs from a fixed class. Let $[n]$ denote $\{1, \ldots, n\}$. For a class $\mathcal{G}$ of graphs, a $d$-fold grid over $\mathcal{G}$ is a graph with vertex set $\left[n_{1}\right] \times \cdots \times\left[n_{d}\right]$ such that each set of vertices where all but one coordinate is fixed induce a graph from $\mathcal{G}$. For example, a Cartesian product of graphs in $\mathcal{G}$ is a $d$-fold grid over $\mathcal{G}$. The family of all $d$-fold grids over $\mathcal{G}$ is denoted by $\mathcal{G}^{d}$.

The study of the chromatic number and independence number of graphs in $\mathcal{G}^{d}$ is related to similar problems appearing in computational geometry. Frequency assignment problems for transmitters in the plane are modeled by coloring and independence problems on certain graphs (see [5]). These graphs arise from sets of points using the Euclidean metric. Analogous problems for the Manhattan metric were addressed in [3]. Since $d-$ fold grids over some classes of graphs can be represented by graphs appearing in this setting, Szegedy [13] posed the following open problem at the workshop "Combinatorial Challenges":

What is the maximum chromatic number of a graph $G \in \mathcal{G}^{d}$ when $\mathcal{G}$ is the class $\mathcal{B}$ of all bipartite graphs or the class $\mathcal{S}$ of graphs containing at most one edge?

If each graph in $\mathcal{G}$ is $k$-colorable, then every graph in $\mathcal{G}^{d}$ has chromatic number at most $k^{d}$, since it is the union of $d$ subgraphs, each of which is $k$-colorable. In particular, all graphs in $\mathcal{B}^{d}$ are $2^{d}$-colorable. We show that this bound is sharp, which is somewhat surprising since Cartesian products of bipartite graphs are bipartite. More generally, let $f(\mathcal{G} ; d)=\max _{G \in \mathcal{G}^{d}} \chi(G)$. We show that if $\mathcal{G}$ is the class of all $k$-colorable graphs, then $f(\mathcal{G} ; d)=k^{d}$. We prove the existence of $k^{d}$-chromatic graphs in $\mathcal{G}^{d}$ probabilistically, but an explicit construction can then be obtained by building, for each $n$, a graph in $\mathcal{G}^{d}$ that is "universal" in the sense that it contains all graphs in $\mathcal{G}^{d}$ with vertex set $[n]^{d}$. This settles the first part of Szegedy's question.

Determining $f(\mathcal{S} ; d)$ is more challenging. Since the maximum degree of a graph in $\mathcal{S}^{d}$ does not exceed $d$, and these graphs do not contain $K_{d+1}$, Brooks' Theorem [2] implies that each graph in $\mathcal{S}^{d}$ is $d$-colorable (when $d \geq 3$ ). Also graphs in $\mathcal{S}^{2}$ are bipartite, since cycles in such a graph alternate between horizontal and vertical edges. In general, graphs in $\mathcal{S}^{d}$ are triangle-free, since any two adjacent vertices differ in exactly one coordinate.

When $d$ is large, we can use a refinement of Brook's Theorem obtained by Reed et al. $[6,9,10,11]$ to improve the upper bound. 
Theorem 1 (Molloy and Reed [10]). There exists a constant $D_{0}$ such that if $D \geq D_{0}$ and $k^{2}+2 k<D$, then every graph $G$ with maximum degree $D$ and $\chi(G)>D-k$ has a subgraph $H$ with at most $D+1$ vertices and $\chi(H)>D-k$.

Theorem 1 implies that $f(\mathcal{S} ; d) \leq d-\sqrt{d}+O(1)$. To see this, observe that any $(d+1)$ vertex subgraph $H$ of a $d$-fold grid over $\mathcal{S}$ has chromatic number at most $d / 2+1$. If $H$ has no vertex with degree at least $d / 2+1 / 2$, then $H$ is $(d / 2+1)$-colorable. If $H$ has a vertex with degree at least $d / 2+1$, then its neighbors form an independent set $A$; since $H-A$ has at most $d / 2$ vertices, the graph $H$ has a proper coloring with $d / 2+1$ colors.

A still better upper bound follows from another result.

Theorem 2 (Johansson [8]). The chromatic number of a triangle-free graph with maximum degree $D$ is at most $O(D / \log D)$.

This result, which was further strengthened by Alon et al. [1], implies that $f(\mathcal{S} ; d) \in$ $O(d / \log d)$, since the neighborhood of every vertex of a $d$-fold grid over $\mathcal{S}$ is independent.

We show that though graphs in $\mathcal{S}^{d}$ are very sparse, and it is natural to expect that they can be colored properly using just a few colors, $f(\mathcal{S} ; d) \geq\left\lfloor\sqrt{\frac{d}{6 \log d}}\right\rfloor$. Our argument is

again probabilistic. A similar argument yields $f(\mathcal{M} ; d) \geq\left\lfloor\frac{d}{6 \log d}\right\rfloor$, where $\mathcal{M}$ is the class of all matchings (i.e., graphs with maximum degree 1$)$. This lower bound is asymptotically best possible, since the discussion above yields $f(\mathcal{M} ; d) \in O(d / \log d)$.

\section{Preliminaries}

In this section, we make several observations used in the proofs of our subsequent lower bounds on $f(\mathcal{G} ; d)$ for various $\mathcal{G}$. We start by recalling the Chernoff Bound, an upper bound on the probability that a sum of independent random variables deviates greatly from its expected value (see [7] for more details).

Proposition 3. If $X$ is a random variable equal to the sum of $N$ independent identically distributed 0,1-random variables having probability $p$ of taking the value 1 , then the following holds for every $0<\delta \leq 1$ :

$$
\operatorname{Prob}(X \geq(1+\delta) p N) \leq e^{-\frac{\delta^{2} p N}{3}} \quad \text { and } \quad \operatorname{Prob}(X \leq(1-\delta) p N) \leq e^{-\frac{\delta^{2} p N}{2}} .
$$

Next, we establish two technical claims. We begin with a standard bound on the number of subsets of a certain size.

Proposition 4. For $\ell, N \in \mathbb{N}$ with $\ell>2$ and $N$ a multiple of $\ell$, the number of $N / \ell$ element subsets of an $N$-element set is at most $2^{\frac{N}{\ell}(1+\log \ell)}$. 
Proof. An $N$-element set has $\left(\begin{array}{c}N \\ N / \ell\end{array}\right)$ subsets of size $N / \ell$. It is well known that $\left(\begin{array}{c}N \\ N / \ell\end{array}\right) \leq$ $2^{N \cdot H(1 / \ell)}$, where $H(p)=-p \log p-(1-p) \log (1-p)$ (see [4], for example). A simple calculation yields the upper bound:

$$
\left(\begin{array}{c}
N \\
N / \ell
\end{array}\right) \leq 2^{N \cdot\left(\frac{1}{\ell} \log \ell+\frac{\ell-1}{\ell} \log \frac{\ell}{\ell-1}\right)} \leq 2^{N \cdot\left(\frac{1}{\ell} \log \ell+\frac{\ell-1}{\ell} \cdot \frac{1}{\ell-1}\right)} \leq 2^{\frac{N}{\ell}(1+\log \ell)}
$$

The second claim is a straightforward upper bound on a certain type of product of expressions of the form $(1-\varepsilon)$ :

Proposition 5. If $a_{1}, \ldots, a_{m}$ are nonnegative integers with sum $n$, then

$$
\prod_{i=1}^{m}\left(1-\left(\begin{array}{c}
a_{i} \\
2
\end{array}\right) \frac{1}{x}\right) \leq\left(1-\frac{1}{x}\right)^{n-m}
$$

for any positive real $x$ such that $x \leq\left(\begin{array}{c}\max _{i} a_{i} \\ 2\end{array}\right)$.

Proof. Since $\sum a_{i}=n$, it suffices to show that

$$
\left(1-\left(\begin{array}{l}
a \\
2
\end{array}\right) \frac{1}{x}\right) \leq\left(1-\frac{1}{x}\right)^{a-1}
$$

for every nonnegative integer $a$. If $a \leq 1$, then the left side of (1) is 1 and the right side is at least 1 . If $a \geq 2$, then (1) follows (by setting $k=a-1$ ) from the well-known inequality

$$
1-\frac{k}{x} \leq\left(1-\frac{1}{x}\right)^{k}
$$

which holds whenever $0 \leq k \leq x$.

\section{Grids over $k$-colorable graphs}

In this section, we prove that $f(\mathcal{B} ; d)=2^{d}$. Note again that after the probabilistic proof of existence, we can construct such graphs explicitly as explained in Section 1. Even so, the argument that they are not $\left(2^{d}-1\right)$-colorable remains probabilistic. Theorem 6 can also be proved by bounding the probability that a random $\left(2^{d}-1\right)$-coloring of some $d$-fold grid over the class of complete bipartite graphs is proper, but we prefer giving a proof via a bound on the size of the largest independent set, since such a bound may be of independent interest.

We prove the result in the more general setting of $k$-colorable graphs. 
Theorem 6. For $d, k \in \mathbf{N}$, there exists a $d$-fold grid $G$ over the class of $k$-colorable graphs such that $\chi(G)=k^{d}$.

Proof. The claim holds trivially if $d=1$, so we assume $d \geq 2$. The integers $k$ and $d$ are fixed, and $N$ is a large integer to be chosen in terms of $k$ and $d$. Consider the set $[N]^{d}$. For each $v \in[N]^{d}$, define a random $d$-tuple $X(v)$ such that $X(v)_{i}$ takes each value in $[k]$ with probability $1 / k$, and the $d$ coordinate variables are independent. Generate a graph $G$ with vertex set $[N]^{d}$ by making two vertices $u$ and $v$ adjacent if they differ in exactly one coordinate and $X(u)_{\ell} \neq X(v)_{\ell}$, where $\ell$ is the coordinate in which $u$ and $v$ differ.

By construction, any set of vertices in $G$ that all agree outside a fixed coordinate induce a complete multipartite graph with at most $k$ parts. Hence $G$ is a $d$-fold grid over the class of $k$-colorable graphs. It will suffice to show that almost surely (as $N$ tends to infinity) $G$ does not have an independent set with more than $\frac{N^{d}}{k^{d}-0.5}$ vertices. This yields $\chi(G) \geq k^{d}$, since otherwise some color class is an independent set of size at least $\frac{N^{d}}{k^{d}-1}$.

For an independent set $A$ in $G$, let the shade of $A$ be the function $\sigma:[d] \times[N]^{d-1} \rightarrow[k]$ defined as follows. For $z=\left(\ell ; i_{1}, \ldots, i_{\ell-1}, i_{\ell+1}, \ldots, i_{d}\right) \in[d] \times[N]^{d-1}$, consider the vertices in $A$ of the form $\left(i_{1}, \ldots, i_{\ell-1}, j, i_{\ell+1}, \ldots, i_{d}\right)$. By the construction of $G$, the value of $X(v)_{\ell}$ is the same for each such vertex $v$, since vertices of $A$ are nonadjacent. Let this value be $\sigma(z)$. If there is no vertex of $A$ with this form, then let $\sigma(z)=1$.

The union of independent sets with the same shade is an independent set. Hence for each function $\sigma$ there is a unique maximal independent set in $G$ with shade $\sigma$; denote it by $A_{\sigma}$. To have $v \in A_{\sigma}$, where $v=\left(i_{1}, \ldots, i_{d}\right)$, the random variables $X(v)_{1}, \ldots, X(v)_{d}$ must satisfy $X(v)_{\ell}=\sigma\left(\ell ; i_{1}, \ldots, i_{\ell-1}, i_{\ell+1}, \ldots, i_{d}\right)$. Hence each $v$ lies in $A_{\sigma}$ with probability $k^{-d}$.

As a result, the expected size of $A_{\sigma}$ is $(N / k)^{d}$. Since the variables $X(v)_{\ell}$ are independent for all $v$ and $\ell$, we can bound the probability that $\left|A_{\sigma}\right| \geq \frac{N^{d}}{k^{d}-0.5}$ using the Chernoff Bound (Proposition 3). Applied with $\delta=\frac{1}{2 k^{d}-1}$, this yields

$$
\operatorname{Prob}\left(\left|A_{\sigma}\right| \geq \frac{N^{d}}{k^{d}-0.5}\right) \leq e^{-\frac{N^{d}}{3\left(2 k^{d}-1\right)^{2} k^{d}}} \leq e^{-\frac{N^{d}}{12 k^{3 d}}} .
$$

Since there are $k^{d N^{d-1}}$ possible shades, the probability that some independent set has more than $\frac{N^{d}}{k^{d}-0.5}$ vertices is at most $k^{d N^{d-1}} \cdot e^{-N^{d} / 12 k^{3 d}}$, and we compute

$$
k^{d N^{d-1}} \cdot e^{-N^{d} / 12 k^{3 d}}=e^{\log k d N^{d-1}-N^{d} / 12 k^{3 d}} \rightarrow 0 .
$$

If $N$ is sufficiently large in terms of $k$ and $d$, then the bound is less than 1 , and there exists such a graph $G$ with no independent set of size at least $\frac{N^{d}}{k^{d}-0.5}$. 


\section{Grids over single-edge graphs}

In this section, we prove the lower bound for $d$-fold grids over the class of graphs with at most one edge.

Theorem 7. For $d \geq 2$, there exists $G \in \mathcal{S}^{d}$ such that $\chi(G) \geq\left\lfloor\sqrt{\frac{d}{6 \log d}}\right\rfloor$.

Proof. Let $k=\left\lfloor\sqrt{\frac{d}{6 \log d}}\right\rfloor$. For $k \leq 2$, the conclusion is immediate. Hence, we assume $k \geq 3$. We generate a graph $G$ with vertex set $[2 k]^{d}$. For $\left(\ell ; i_{1}, \ldots, i_{\ell-1}, i_{\ell+1}, \ldots, i_{d}\right) \in$ $[d] \times[2 k]^{d-1}$, choose a random pair of distinct integers $j$ and $j^{\prime}$ from $[2 k]$, and make the vertices $\left(i_{1}, \ldots, i_{\ell-1}, j, i_{\ell+1}, \ldots, i_{d}\right)$ and $\left(i_{1}, \ldots, i_{\ell-1}, j^{\prime}, i_{\ell+1}, \ldots, i_{d}\right)$ adjacent in $G$. The choices of $\left\{j, j^{\prime}\right\}$ are independent for all elements of $[d] \times[2 k]^{d-1}$.

Since $G \in \mathcal{S}^{d}$, its chromatic number is at most $d$. To show that the event $\chi(G) \geq$ $k$ has positive probability, it suffices to show that with positive probability, $G$ has no independent set of size at least $(2 k)^{d} / k$.

Consider a set $A$ in $V(G)$ with size $(2 k)^{d} / k$; we bound the probability that $A$ is an independent set in $G$. Again we think of an element $z$ in $[d] \times[2 k]^{d-1}$ as designating a line in $[2 k]^{d}$ parallel to some axis. Let $A[z]$ be the intersection of $A$ with this line. By the construction of $G$, the probability that no two vertices in $A[z]$ are adjacent in $G$ is

$$
1-\left(\begin{array}{c}
|A[z]| \\
2
\end{array}\right) /\left(\begin{array}{c}
2 k \\
2
\end{array}\right)
$$

which is at most $1-\left(\begin{array}{c}|A[z]| \\ 2\end{array}\right) \frac{1}{2 k^{2}}$. By applying Proposition 5 with $x=1 / 2 k^{2}, n=|A| \geq$ $\frac{(2 k)^{d}}{k}=2(2 k)^{d-1}$, and $m=(2 k)^{d-1}$, we conclude that the probability of all subsets of $A$ lying along lines in a particular direction being independent in $G$ is at most

$$
\prod_{z \in\{\ell\} \times[2 k]^{d-1}}\left(1-\left(\begin{array}{c}
|A[z]| \\
2
\end{array}\right) \frac{1}{2 k^{2}}\right) \leq\left(1-\frac{1}{2 k^{2}}\right)^{(2 k)^{d-1}} .
$$

Let $p$ be the probability that $A$ is an independent set in $G$. Since the edges in each of the $d$ directions are added to $G$ independently,

$$
p \leq\left(1-\frac{1}{2 k^{2}}\right)^{d(2 k)^{d-1}} \leq e^{-\frac{d(2 k)^{d-1}}{2 k^{2}}} \leq e^{-2 d(2 k)^{d-3}} \leq 2^{-2 d(2 k)^{d-3}}
$$

We want to show that with positive probability, $G$ has no independent set of size $(2 k)^{d} / k$. Let $M$ be the number of subsets of $V(G)$ with size $(2 k)^{d} / k$. By Proposition 4 ,

$$
M \leq 2^{\frac{(2 k)^{d}}{k} \cdot(1+\log k)} \leq 2^{2(2 k)^{d-1} \cdot(1+\log k)} \leq 2^{3(2 k)^{d-1} \log k} .
$$


Therefore, we bound the probability that $G$ has an independent set of size $(2 k)^{d} / k$ by the following computation:

$$
2^{3(2 k)^{d-1} \log k} \cdot 2^{-2 d(2 k)^{d-3}}=2^{2(2 k)^{d-3}\left(6 k^{2} \log k-d\right)}<1 .
$$

The last inequality uses the fact that $6 k^{2} \log k-d$ is negative, by the choice of $k$. We conclude that some such graph $G$ has no independent set of size at least $(2 k)^{d} / k$.

\section{Grids over matchings}

Finally, we consider $d$-fold grids over the class $\mathcal{M}$ of matchings.

Theorem 8. For $d \geq 2$, there exists $G \in \mathcal{M}^{d}$ such that $\chi(G) \geq\left\lfloor\frac{d}{6 \log d}\right\rfloor$.

Proof. As the proof is similar to the proof of Theorem 7, we will give less detail and focus on the differences between the proofs. Set $k=\left\lfloor\frac{d}{6 \log d}\right\rfloor$ and assume $k \geq 3$. We randomly generate a graph $G$ with vertex set $[2 k]^{d}$. As before an element $z$ in $[d] \times[2 k]^{d-1}$ designates a line in $[2 k]^{d}$ parallel to some axis. We place a random perfect matching on the $2 k$ vertices in each such line. Hence, the resulting graph $G$ is $d$-regular. It suffices to show that with positive probability, $G$ has no independent set of size at least $(2 k)^{d} / k$.

Consider a set $A$ in $V(G)$ with size $(2 k)^{d} / k$; we bound the probability that $A$ is an independent set in $G$. Let $A[z]$ be the intersection of $A$ with a line designated by $z \in[d] \times[2 k]^{d-1}$. Let $X$ be the random variable that is the number of edges in $G$ induced by $A[z]$. By the construction of $G$, we have $\mathrm{E}(X)=\frac{1}{2 k-1}\left(\begin{array}{c}|A[z]| \\ 2\end{array}\right)$. When $X$ is a nonnegative integer-valued random variable, $\operatorname{Prob}[X \geq 1] \geq \frac{\mathrm{E}(X)}{\max (X)}$. Since $A[z]$ cannot induce more than $|A[z]| / 2$ edges, we obtain a lower bound on the probability $p$ that $A[z]$ contains an edge by computing

$$
p \geq \frac{\frac{1}{2 k-1}\left(\begin{array}{c}
|A[z]| \\
2
\end{array}\right)}{|A[z]| / 2}=\frac{|A[z]|-1}{2 k-1} \geq \frac{|A[z]|-1}{2 k} .
$$

Let $q_{\ell}$ denote the probability that all subsets of $A$ lying along lines in direction $\ell$ are independent (each such line consists of $d$-tuples that agree outside the $\ell$ th coordinate). We compute

$$
\begin{gathered}
q_{\ell} \leq \prod_{z \in\{\ell\} \times[2 k]^{d-1}}\left(1-\frac{|A[z]|-1}{2 k}\right) \leq \prod_{z \in\{\ell\} \times[2 k]^{d-1}} e^{-\frac{|A[z]|-1}{2 k}} \\
=e^{-\frac{(2 k)^{d} / k-(2 k)^{d-1}}{2 k}}=e^{-(2 k)^{d-2}} .
\end{gathered}
$$

The probability $P$ that $A$ is independent can now be bounded as follows:

$$
P \leq e^{-d(2 k)^{d-2}} \leq 2^{-d(2 k)^{d-2}}
$$


Finally, an upper bound on the probability that $G$ has an independent set of size $(2 k)^{d} / k$ is obtained by multiplying the bound on $P$ and the bound on the number of $(2 k)^{d} / k$-element subsets of vertices from Proposition 4.

$$
2^{3(2 k)^{d-1} \log k} \cdot 2^{-d(2 k)^{d-2}}=2^{(2 k)^{d-2}(6 k \log k-d)}<1 .
$$

\section{Open problem}

We determined assymptotically the function $f(\mathcal{G} ; d)$ for the class $\mathcal{G}$ of $k$-colorable graphs and the class $\mathcal{M}$ of matchings. For the class $\mathcal{S}$ of graphs with at most one edge, we were not able to obtain matching lower and upper bounds. Our results imply only that

$$
\left\lfloor\sqrt{\frac{d}{6 \log d}}\right\rfloor \leq f(\mathcal{M} ; d) \leq O\left(\frac{d}{\log d}\right) .
$$

Hence, it remains open to determine the assymptotic behavior of the function $f(\mathcal{M} ; d)$ in terms of $d$.

\section{Acknowledgement}

This research was conducted during the DIMACS, DIMATIA and Renyi tripartite workshop "Combinatorial Challenges" held in DIMACS in April 2006; we thank the DIMACS center for its hospitality. We also thank Stephen Hartke and Hemanshu Kaul for participating in lively early discussions at DIMACS about the problem.

\section{References}

[1] N. Alon, M. Krivelevich, B. Sudakov: Coloring graphs with sparse neighborhoods, J. Combinatorial Theory Ser. B 77(1) (1999), 73-82.

[2] R. L. Brooks: On coloring of the nodes of a network, Proc. Cambridge Phil. Soc. 37 (1941), 194-197.

[3] X. Chen, J. Pach, M. Szegedy, G. Tardos: Delaunay graphs of point sets in the plane with respect to axis-parallel rectangles, in: Proc. 19th annual ACM-SIAM symposium on Discrete algorithms (SODA) 2008, 94-101.

[4] T. M. Cover, J. A. Thomas: Elements of information theory, John Wiley \& Sons, 1991. 
[5] G. Even, Z. Lotker, D. Ron, S. Smorodinsky: Conflict-free colorings of simple geometric regions with applications to frequency assignment in cellular networks, SIAM J. Comput. 33 (2003), 94-136.

[6] B. Farzad, M. Molloy, B. Reed: $(\Delta-k)$-critical graphs, J. Combinatorial Theory Ser. B 93 (2005), 173-185.

[7] T. Hagerup, Ch. Rüb: A guided tour of Chernoff bounds. Inform. Process. Letters 33 (1989) 305-308.

[8] A. R. Johansson: Asymptotic choice number for triangle-free graphs, manuscript.

[9] M. Molloy, B. Reed: Colouring graphs whose chromatic number is near their maximum degree, in: Proc. 3rd Latin American Symposium on Theoretical Informatics (LATIN) 1998, 216-225.

[10] M. Molloy, B. Reed: Colouring graphs when the number of colours is nearly the maximum degree, in: Proc. 33rd annual ACM symposium on Theory of computing (STOC) 2001, 462-470.

[11] B. Reed: A strengthening of Brooks' theorem, J. Combinatorial Theory Ser. B 76 (1999), 136-149.

[12] G. Sabidussi: Graphs with given group and given graph-theoretical properties, Canad. J. Math 9 (1957), 515-525.

[13] M. Szegedy, presentation at open problem session of DIMACS, DIMATIA and Renyi tripartite workshop "Combinatorial Challenges", DIMACS, Rutgers, NJ, April 2006. 